SCIENTIFIC REPORT

\title{
An experimental study on the effect of encircling band procedure on silicone oil emulsification
}

\author{
D J de Silva, K S Lim, W E Schulenburg
}

Br J Ophthalmol 2005;89:1348-1350. doi: 10.1136/bjo.2004.063768

Aim: Silicone oil is a useful tamponading material used in complex vitroretinal surgery. However, the use of silicone oil is associated with emulsification which can lead to vision threatening complications. The authors developed an experimental model to study the effect of encircling band on silicone oil emulsification.

Methods: Two identical artificial eye chambers were constructed with circumferential indentations placed at the sphere's equator (mimicking an encircling band indentation), and filled with varying amounts of Silicone Oil 1000 centistrokes (Adato, Bausch and Lomb, UK) and balanced salt solution. The chambers were then placed on a horizontal rotating shaker, mimicking physiological saccadic eye movements, which spun the chambers at $100 \mathrm{~Hz}$ for 5 days at $37^{\circ} \mathrm{C}$. Emulsification was then quantified by dark field microscopy, digital photography, and manual counting by a masked observer.

Results: The mean (standard deviation (SD)) values of silicone emulsification bubbles were as follows: in the $90 \%$ silicone oil filled chamber with no encircling band, 139.1 (SD 313.4); in the $90 \%$ silicone oil filled chamber with encircling band, 10.9 (SD 22.2) ( $<<0.0001)$; in the $75 \%$ silicone oil filled chamber with no encircling band, 103.6 (SD 272.6); in the $75 \%$ silicone oil filled chamber with encircling band, 18.5 (SD 32), $(p=0.001)$.

Conclusions: The emulsification of silicone oil results from friction between the silicone oil and aqueous liquids. The results from this study suggest silicone oil emulsification is reduced by (1) more complete silicone oil fill and (2) indentation from an encircling band. The authors hypothesise that both these measures resulted in reduced emulsification by reducing silicone oil/aqueous movement and resulting shearing forces.

$\mathrm{T}$ he management of complicated retinal detachment is frequently facilitated by the use of intravitreal materials that assist with retinal reapposition. Silicone oil is a useful tamponading material used in complex vitroretinal surgery. However, the use of silicone oil is associated with emulsification which can lead to vision threatening complications such as silicone keratopathy and secondary glaucoma. $^{1-3}$ We developed an experimental model using an artificial eye chamber and mechanical shaker to assess whether the use of an encircling band or varying levels of silicone oil fill would effect silicone oil emulsification.

\section{METHODS}

Two identical artificial eye chambers were constructed using clear perspex. Each sphere was constructed of two perspex halves that were sealed together with four bolts (fig 1) Each chamber had a spherical volume of $4 \mathrm{ml}$ and a single access port to allow easy filling of the chamber. One model had a circumferential band placed at the sphere's equator that mimicked an encircling band indentation height of $1.5 \mathrm{~mm}$. One chamber with no encircling band was filled with $90 \%$ Silicone Oil 1000 centistrokes (Adato, Bausch and Lomb, UK) and $10 \%$ balanced salt solution, this is considered to be the control. A second chamber with an encircling band was filled with $90 \%$ silicone oil and $10 \%$ balanced salt solution. The experiment was repeated with the two chambers filled with $75 \%$ silicone oil. Syringes were used to inject the liquids into the eye chamber and care taken to avoid mixing of the liquids or the introduction of air bubbles. The two liquids formed two immiscible phases when injected into the chambers, with the silicone oil forming the upper phase. The two eye chambers with the encircling band positioned in the vertical meridian were then placed on a horizontal rotating shaker. The shaker mimicked physiological saccadic eye movements and spun the chambers at $100 \mathrm{~Hz}$ for 5 days at $37^{\circ} \mathrm{C}$. All experiments were repeated five times. An emulsifying agent was not used; however the eye chambers were immediately opened and the contents emptied, thereby not allowing sufficient time for the two mediums to separate. The order of the opening with and without an encircling band was alternated. The extent of emulsification was then quantified by dark field microsopy, using a magnification factor of $\times 10$. Ten random digital photographs (fig 2) were taken in the central field of view, quantification was done by manual counting of emulsification bubbles by a masked observer, and the mean number of emulsification bubbles for each individual eye chamber was calculated, using a previously described technique. ${ }^{4}$

\section{RESULTS}

Examples of the dark field photographs from the silicone oil with emulsification are shown in figure 2. Results are shown in table 1 and figure 3. The Mann Whitney non-parametric test was used to analyse the results; $90 \%$ silicone oil filled chamber with no encircling band was the control.

Emulsification was significantly reduced in the eye chambers with an encircling band in both the $90 \%$ and $75 \%$ silicone oil filled models. The mean of emulsification bubbles were also significantly lower in the $90 \%$ oil filled with encircling band compare to $75 \%$ oil filled with encircling band $(\mathrm{p}=0.001)$.

\section{DISCUSSION}

Silicone oil emulsification is a key factor in the complications of silicone oil use as an intraocular tamponade. Previous studies have investigated physiochemical properties of the silicone oil and their role in emulsification. The purpose of our experiment was to assess physical factors that may reduce silicone oil emulsification in vitro. We compared silicone oil emulsification in two identical eye chambers assessing the use of an encircling band and a more complete fill of silicone oil. 

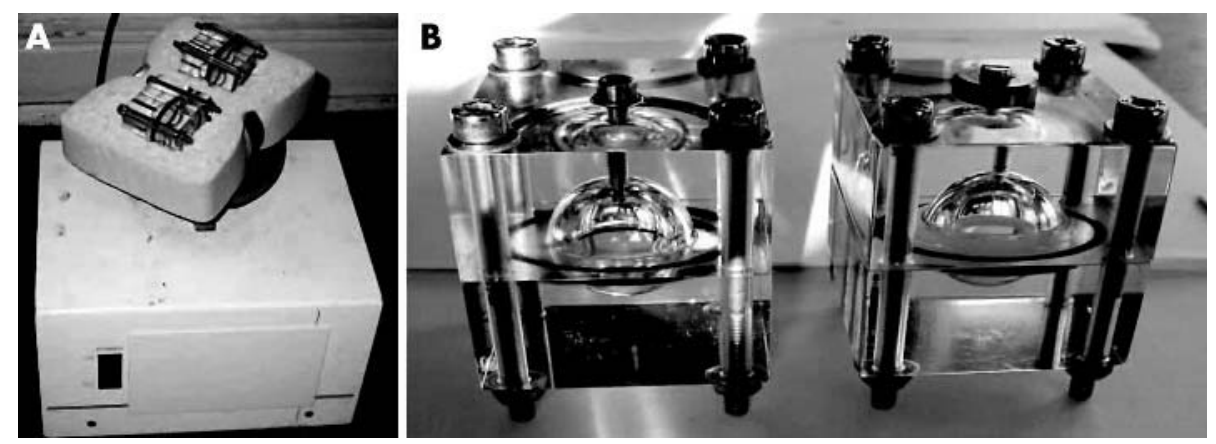

Figure 1 (A) Two artificial eye chambers placed on a horizontal rotating shaker. (B) Two artificial eye chambers; the right chamber contains a circumferential plastic band placed at the sphere's equator which mimicked encircling band indentation.

Silicone keratopathy, cataract formation, and silicone retinopathy have been attributed with silicone oil toxicity and their physiochemical properties. ${ }^{25}$ Silicone oil droplets generated by emulsification may enter the anterior chamber through an intact zonular framework and droplets have been found in the trabecular meshwork, iris, ciliary body, retina, and optic nerve. ${ }^{67}$ Silicone oil droplets can enter the anterior chamber angle and may lead to refractory glaucoma. Vision threatening complications may result from silicone oil emulsification and may occur over a spectrum of the postoperative period, removal of the oil may not reverse its toxic effects. ${ }^{3}$ The mechanism of silicone oil induced toxicity remains unclear. ${ }^{5}$

Previous studies have found multiple factors effecting the emulsification of silicone oil: viscosity of silicone oil, surface tension agents, and fraction of silicone oil. ${ }^{4}$ Emulsification is defined as a dispersion of fine liquid particles in another liquid medium and results from shearing forces between the two mediums, causing droplets to be pinched off into the other medium due to surface tension. Droplets start at the interface of the two liquids and give rise to an emulsion. For the emulsification to progress, kinetic energy is required and may be counteracted by surface agent stabilisers. ${ }^{5}$ The process undergoes three stages-creaming, flocculation, and coalescence-and is characterised by the accumulation of fine oil droplets on the surface of the intraocular fluids. The process of coalescence results in a variation of droplet size and is aided in vivo by the presence of emulsifiers. ${ }^{8-10}$ Low molecular weight components such as phospholipids, proteins, and ions are likely to influence the emulsification process, however their role in vivo is not known. ${ }^{4}$ Our results were consistent with previous models of silicone oil emulsification: the bubbles were stable, demonstrated regular borders, and
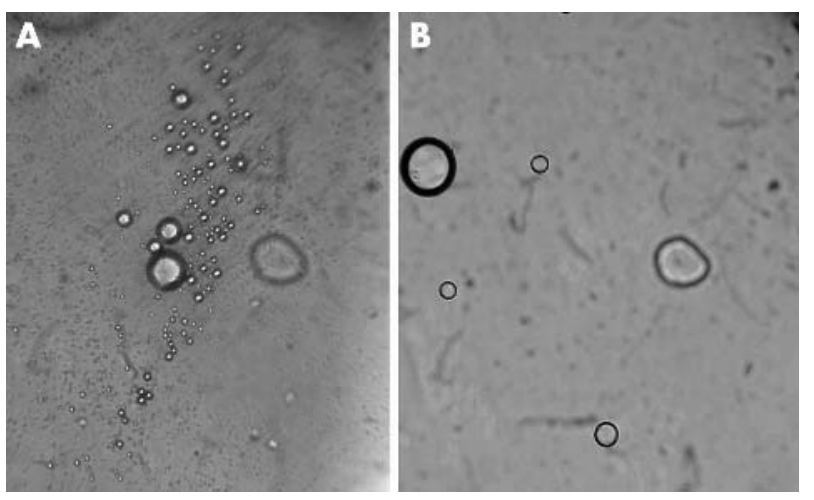

Figure 2 Dark field photomicrographs $(\times 10)$ demonstrating effects of encircling band procedure on silicone oil emulsification. (A) Silicone oil bubbles of the $90 \%$ silicone oil filled eye chamber without encircling band. (B) Silicone oil bubbles of the $90 \%$ silicone oil filled chamber with encircling band.
Table 1 Silicone emulsification in the eye chambers with and without encircling band

\begin{tabular}{lcc}
\hline & $\begin{array}{l}\text { Silicone oil emulsification, } \\
\text { mean (SD) }\end{array}$ & p Value* \\
\hline $\begin{array}{l}90 \% \text { silicone oil filled without } \\
\text { encircling band }\end{array}$ & $139.1(313.4)$ & $<0.0001$ \\
$\begin{array}{l}90 \% \text { silicone oil filled with } \\
\text { encircling band }\end{array}$ & $10.9(22.2)$ & 0.001 \\
$\begin{array}{l}75 \% \text { silicone oil filled without } \\
\text { encircling band } \\
75 \% \text { silicone oil filled with } \\
\text { encircling band }\end{array}$ & $103.6(272.6)$ & 0.039 \\
\hline *Mann-Whitney non-parametric test. & \\
\hline
\end{tabular}

showed a variation in size. ${ }^{4}$ Emulsification occurs at the interface between the silicone oil and aqueous layers as a consequence of shearing forces. In vivo, some aqueous fluid is likely to line the wall of the eye and emulsification may occur both at the wall and at the silicone oil/aqueous interface.

A number of studies have looked at the use of experimental eye chambers and the use of silicone oil as a tamponading agent. ${ }^{11-15}$ Perspex, a hydrophilic material, is a material which mimics the surface properties of the retina. In addition, the use of a protein solution to coat the surface of the artificial
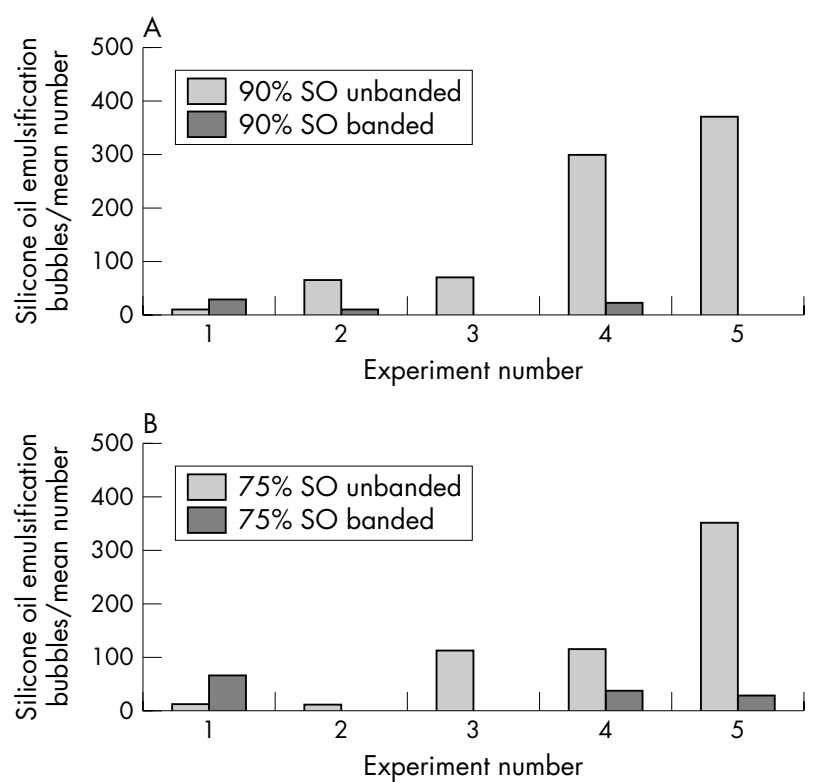

Figure 3 Graphs showing silicone oil emulsification in the eye chambers with and without encircling band. (A) $90 \%$ silicone oil filled. (B) $75 \%$ silicone oil filled. 
eye chamber has been found to further represent the contact of silicone oil to the retina in vivo. In this experiment we chose not to coat the chamber with protein-a layer of protein may not impact on emulsification as the interface of a thin film of aqueous solution may not deform sufficiently to cause emulsification into the film. In addition, shaking the chambers at $100 \mathrm{~Hz}$ may cause the protein layer to detach from the artificial chamber walls. We believe our model is valid, and offers some insight into the effects of encircling band procedure on silicone oil emulsification.

The results from this study suggest two important factors in the process of emulsification of silicone oil and how this process may be reduced: (1) the more complete the silicone oil fill in the eye chamber, the lower the level of silicone oil emulsification and (2) the indentation from the encircling band exerted a stabilising effect on the silicone oil globule during eye movement resulting in reduced shearing forces and silicone oil emulsification.

The results of our experiment may be explained by the physical properties of silicone oil and its interaction with the artificial eye chamber. The contact angle between the silicone oil and the artificial eye chamber is small, and to induce an $180^{\circ}$ tamponade, an $80 \%$ fill is estimated. ${ }^{11}$ It follows that with an increased silicone oil fill, the total area of contact between silicone oil and the surrounding aqueous layer is increased. With an increased silicone oil fill we propose that the increased size of the silicone oil bubble resulted in reduced movement of the silicone oil and aqueous layers. This resulted in an increased stability, a reduction of shearing forces, and silicone oil emulsification.

Encircling scleral buckle procedure induces a geometric alteration in the anatomy of the eye. This configurational change influences both axial length and induces indentation of the globe resembling an hourglass shape. ${ }^{12}{ }^{13}$ The influence of an encircling band in our artificial model eye resulted a significant reduction of silicone oil emulsification. We hypothesise that the encircling band leads to reduced emulsification by reducing silicone oil/aqueous movement and resulting shearing forces.

Silicone oil emulsification can lead to vision threatening complications and removal of the oil may not reverse these changes. Our in vitro experiments suggest an encircling band procedure and increased silicone oil fill may result in reduced silicone oil emulsification.
Authors' affiliations

D J de Silva, K S Lim, W E Schulenburg, Western Eye Hospital,

Marylebone Road, London, UK

K S Lim, Ocular Repair and Regeneration Biology Unit and Department of Pathology, Institute of Ophthalmology, London, UK

Sponsor details: none.

Competing interests: none declared

Correspondence to: Mr W E Schulenburg, Consultant in

Ophthalmology, Western Eye Hospital, Marylebone Road, London

NWI 5YE, UK; drdjdesilva@yahoo.co.uk

Accepted for publication 18 April 2005

\section{REFERENCES}

1 Leaver PK, Grey RHB, Garner A. Complications following silicone injection. Mod Probl Ophthalmol 1979;20:290-4.

2 Gabel VP, Kampik A, Burkhardt J. Analysis of intraocularly applied silicone oils of various origins. Graefes Arch Clin Exp Ophthalmol 1987;225:160-2.

3 Laqua $\mathrm{H}$, Lucke K, Foerster MH. Development and current status of silicone oil surgery. Klin Monatsbl Augenheilkd 1988;192:277-83.

4 Crisp A, de Juan E Jr, Tiedeman J. Effect of silicone oil viscosity on emulsification. Arch Ophthalmol 1987;105:546-50.

5 Heidenkummer HP, Kampik A, Thierfelder S. Emulsification of silicone oils with specific physicochemical characteristics. Graefes Arch Clin Exp Ophthalmol 1991;229:88-94.

6 Valone J Jr, McCarthy M. Emulsified anterior chamber silicone oil and glaucoma. Ophthalmology 1994;101:1908-12.

7 Kirchhof B, Tavakolian U, Paulmann H, et al. Histopathological findings in eyes after silicone oil injection. Graefes Arch Clin Exp Ophthalmol 1986;224:34-7.

8 Nakamura K, Refojo MF, Crabtree DV. Factors contributing to the emulsification of intraocular silicone and fluorosilicone oils. Invest Ophthalmol Vis Sci 1990;31:647-56.

9 Becher P, ed. Encyclopedia of emulsion technology, Vol 2: Applications. New York: Marcel Dekker, 1985:1-69.

10 Sherman P. Emulsion science. London: Academic Press, 1968:59-65

11 Fawcett IM, Williams RL, Wong D. Contact angles of substances used for internal tamponade in retinal detachment surgery. Graefes Arch Clin Exp Ophthalmol 1994;232:438-44.

12 Wong D, Williams RL, German MJ. Exchange of perfluorodecalin for gas or oil: a model for avoiding slippage. Graefes Arch Clin Exp Ophthalmol 1998;236:234-7.

13 Williams R, Wong D. The influence of explants on the physical efficiency of tamponade agents. Graefes Arch Clin Exp Ophthalmol 1999;237:870-4.

14 Herbert E, Stappler T, Wetterqvist C, et al. Tamponade properties of doublefilling with perfluorohexyloctane and silicone oil in a model eye chamber. Graefes Arch Clin Exp Ophthalmol 2004;242:250-4.

15 Wetterqvist C, Wong D, Williams R, et al. Tamponade efficiency of perfluorohexyloctane and silicone oil solutions in a model eye chamber. Br J Ophthalmol 2004;88:692-6. 